\title{
Protein Scribble Homolog
}

National Cancer Institute

\section{Source}

National Cancer Institute. Protein Scribble Homolog. NCI Thesaurus. Code C125584.

Protein scribble homolog (1630 aa, $175 \mathrm{kDa}$ ) is encoded by the human SCRIB gene. This protein is involved in cell polarization. 